\title{
CINCO PAPELETAS BIBLIOGRÁFICAS SOBRE LOPE DE VEGA
}

Gracias a los desvelos de $\mathrm{F}$. Zamora Lucas ${ }^{1}$ se han podido reunir las poesías que Lope - por compromiso amistoso- puso al frente de algunos libros ajenas. Con el núm. 51, el compilador recoge el primer verso de una décima y añade luego: «Existía el libro, en 1935, en la Biblioteca de la Facultad de Filosofía y Letras de Madrid. No hemos podido localizarlo después de esa fecha» (p. 44) ${ }^{2}$. El mismo investigador ${ }^{3}$, con una encomiable honestidad científica, señala no haber visto otro libro en el que Lope colaboró ${ }^{4}$. En ambos libros se encuentran ciertos datos que pueden interesar a los futuros estudiosos del «Monstruo de la Naturaleza»; por eilo me permitiré, líneas después, hacer una exacta descripción de tales obras.

'También se debe a la diligencia del Sr. Zamora la publicación Lope de Vega, censor de libros ${ }^{5}$. De todo el material inventariado $-\mathrm{y}$ es muchísimo- sólo cinco obras han escapado a la paciente búsqueda del erudito bibliotecario. Dos de ellas han sido vistas por mí y daré la descripción. Sin embargo, las referencias que se poseen de una de ellas son -al parecerfalaces; no obstante, describiré el ejemplar que he tenido en mis manos porque acaso pueda servir a futuros investigadores.

En quinto lugar describo otro libro raro en el que se hace referencia a Lope. Creo que bien valdrá unir los Favores de las Musas a este parvo espigueo.

1 LoPe DE VEGa: Poesias preliminares de libros, Madrid, Cuadernos bibliográficos del C. S. I. C., II, 1961.

2 En efecto, tal procedencia consta en el Catálogo de la exposición bibliográfica de Lope de Vega organizada por Bibliotcca Nacional, Madrid, 1935, p. 226, núm. 862. Véase también Zamora Lucas, Lope de V $\mathrm{cga}$, censor de libros, citado después, p. 81.

- Núm. 44, p. 41.

- Sin embargo copia, y con fidelidad, el poema laudatorio de nuestro gran dramaturgo.

"Larach:", Artes Gráficas Bescá, 1941.

30 
Durante los meses de mayo y junio de 1962 pude trabajar en la Biblioteca de la Hispanic Society de Nueva York: una ayuda de investigación de la Universidad de California -donde entonces profesaba- me permitió recurrir a los inmensos fondos bibliográficos de las grandes bibliotecas del Este de los Estados Unidos. Aunque mi interés entonces era totalmente distinto del que motiva estas notas, pude buscar una veintena de libros raros que nunca había conseguido ver. Trece de ellos estaban en la Hispanic Society y su acceso me fue cómodo y fácil, gracias a la gentileza de los directivos de la Sociedad y de los técnicos de la Biblioteca. Conste - en esta modestísima aportación - mi gratitud a la Universidad de California y a la Hispanic Society.

A continuación ordeno las papeletas bibliográficas ${ }^{1}$. No me limitaré a las simples referencias, sino que, cuando baya algún dato de interés, lo transcribiré para hacer asequible obras tan llenas de rareza.

1. Zamora Lucas (Poesías preliminares) ha publicado cuatro poemas de Lope en honor del Licenciado Francisco de Herrera (vid. sus números 4447, págs. 40-42); en las líneas que siguen no voy a repetir lo ya sabido, sino a describir una obra, al parecer, muy poco manejada ${ }^{2}$. Las dimensiones

1 Ninguna de ellas ticne cabida en la breve muestra Suplemento a los varios versos $y$ prosas de Lope de Vega Carpio, que se hallan esparcidos en algunos libros en alabanza de ellos y de sus autores, que ocupa las págs. 167-192 del t. XXI de la Colección de las Obras sueltas que ordenó Cerdí y Rico. Madrid, Imprenta de Sancha, 1779. Tampoco figuran en las Obras varias de Lope de Vega Carpio sacadas de varios autores a quienes él elogió, inventario anónimo del S. XVIII cuyo índice consta en RICARDo Del ARco, La sociedad española en las obras dramáticas de Lope de Vega, Madrid, 1941, p. $673 a-b$. E1 manuscrito (con el núm. 278) figura en la p. 67 del Catálogo de la exposición bibliográfica, citado en la nota 2.

2 Juan Millé y Giménez, Apuntes para una bibliografía de las obras no dramáticas atribuidas a Lope de Vega, BHi, 1928, LXXIV, p. 387, bajo su núm. 73 facilita la siguiente referencia: "HERRERA MALDONADO (LICENCIADO DON FRANCISCO DE ). Sanazaro español, Madrid, 1920. Obra dedicada a Lope Poesía de éste en loor de Herrera Maldonado (PÉrez Pastor, B. M., II, núm. 1.697; Gallardo, Ensayo, III, volúmenes 205-206)». Ambos bibliófilos describieron el libro, pero no copiaron el texto de Lope.

En la Silva II del Laurel de Apolo, Lope dedicó a su amigo Herera Maldonado el siguiente elogio:

Preciada de las musas Oropesa,

Dijo que en el Parnaso graduado

Don Francisco de Herrera Maldonado,

Había de ser el héroe desta empresa;

Porque si España de alabar no cesa

Sus versos y su prosa,

Ellos dulces y graves y ella hermosa, 
del libro son 14.2 cms. de alto por 9.6 de ancho. Consta de t9 tolios. I a distribución de la portada es como sigue:

SANAZARO / Español / Los tres libros / del Parto de la Virgin / nuestra Señora. / TRADVCCION CASTELLANA / de verso Heroyco Latino. / Por el Licenciado don / Francisco de Herrera Maldonado. Canónigo de / la santa Yglesia Real de Arbas de Leon, / y natural de la ailla de Oropesa. / A LOPE DE VEGA C.ARPIO, / Fiscal de su Santidad en su Ca- / mara Apostolica. / con privilegio. / En Madrid, por Fernando Corred de Mon- / tenegro, Año de 1620. / A costa de Andres de Carrasquilla. / Vendese en la calle mayor, y en Palacio.

E1 contenido de la obra es el siguiente:

f. 2r. Aprobación del P. Juan de Monroy, de la Compañía de Jesús.

f. $2 v$. Aprouación del Padre Maestro Fr. / Ortensio Felix Paranicino, Predicador de su Magestad, y Pro- / uincial de la Orden de la / Santissima Tri- / nidad.

Por Mandado de V. A. he visto la traduc- / cion que el Licenciado don Francisco de He- / rrera Maldonado, Canonigo de la Santa / Yglesia de Arbas ha hecho en nuestra lengua / Castellana, del libro de Sanazaro. que intitulo / de Partu Virginis, y el libro ha sido tan justa- / mente celebrado con publica, y vniversal acla- / mación de todas lás naciones que le deue muchis- / simo al tradućtor la vuestra, por auerla queri- / do enriquezer con este tesoro, con que glorio- / samente nos ha honrado: porque la materia, el estilo, la doctrina, todo merece gozar de la luz comun $y$ de la medced que V. A. le hiziere con su licencia.

Fr. Ortensio Felix / Parauicino.

f. 5r-5v [sin numerar] DE LOPE DE VEGA / Carpio, al Licenciado Don Fran- / cisco de Herrera Mal- / donado.

[5r]

Los Espiritus puros,

Que al transito asistieron soberano

Del Verbo, al velo humano,

$Y$ de Dauid en los neuados muros,

Vieron temblando al yelo,

En braços de la Aurora, al sol del cielo.

A ning no negar le competía,

Concedierun la historia y la poesía,

$Y$ a la envidia cruel, que no se excusa,

Mostraron el espejo de Medusa.

(BAAEE, XXXVIII, p. 196 b.)

A él le dedico, también, la hermosa epístola en que narra la profesión de Marcela 
Aquellos que adoraron,

I.a humanidad que despreció el lucero,

$\mathrm{Y}$ en extasis miraron

Los primeros singultos del cordero,

Oyendo en mortal nombre,

Al gran Hijo de Dios, suspiros de hombre.

Celebren, docto Herrera,

Y premien Sanaçaro Castellano,

Del sol la nueua esfera,

El nueuo altar de nieue, que tu mano,

$Y$ ilustre pluma, en verso,

De España erige al Rey del vniuerso.

Que las Musas profanas,

No tienen para dar a sus ${ }^{1}$ pinceles,

En sus pimpleas vanas

[jv] Augustas palmas, Daphnicos laureles,

Que a tu sacra pintura

Es menos que del sol, corona escura.

Como forman estrellas,

De las constelaciones celestiales

Las simitrias bellas,

Assi con los diamantes Orientales,

La Lira forme el cielo,

Con que cantas a Dios en mortal velo.

Que mientras el vndoso

Tajo siguiere su inmortal camino

(Francisco Generoso),

El nueuo nombre de Amphion diuino,

Con que a la fama llegas

Celebraran las Musas en sus Vegas.

$Y$ diran que tu solo

Quitaste de su Templo soberano

(Como segundo Apolo),

La Lira del pastor Napolitano,

$Y$ que fue tan diuino

Tu canto, que ygualò con el Latino.

Hay otros elogios del Conde de Lodron, de D. Jerónimo de Herrera y Guzmán, de D. Pedro de Herrera Maldonado, de Juan de Melo Carrillo, del Ledo. Francisco de Córdoba, de D. Bernardino Manrique de Ayala, de Alonso Gerónimo de Salas Barbadillo, de Alfonso Ribero Pegado, de Andrés de Carrasquilla, del P. Juan de Monroy, S. J., del P. Fr. Manuel de Jesús María, carmelita descalzo, del P. M. ${ }^{\circ}$ Fr. Lorenço Gracián de la Madre de Dios y del Lcdo. Francisco Martínez Portichuelo.

2r-2v. A Lope de Vega Carpio.

$3 r-8 r$. Prólogo al lector.

f. $1 r-79 q^{\prime}$. Texto. [Frente al f. 1, viñeta tosca de la Virgen sobre la luna.]

1 Error por tus. 
2. La Justa poética que voy a describir fue ignorada en los Apuntes, de Millé, y es uno de esos cinco libros que huyeron a las pesquisas de $\mathrm{Za}$ mora, y de los que dijo que, son «de tal rareza, que casi pudiéramos dar ya como desaparecido» ${ }^{1}$. El opúsculo tiene 40 folios y mide 14,3 cms. de alto por 9,2 de ancho.

Ivsta / POET I C A, / gue hizo al santis-/simo sacramento en / La villa de CIFVENTES, el / DOTOR IVAN GVTIERREz Me-/Dico DE SV Magestad. / Recopilada por iego Manuel, Capellán de Don / Iuan de Tapia / Dirigida al mismo Dotor. / [Viñeta de un clérigo, que lleva una custodia, y va revestido con capa pluvial; a sus lados, sendos acólitos con cirios. La viñeta va rodeada por las siguientes frases latinas:1 Venite ad me omnes, qui laboratis / \& onerati estis, \& ego / reficiam vos. Matth. II. cap. / En Madrid en la Imprenta Real. Año de 1621.

f. 2r. Suma de la tassa [fechada el 27 de mayo de 1621, ante Lázaro de los Ríos]. Fe de erratas por el Licenciado Murcia de la Llana.

i. 2v. Suma del privilegio [ante Lázaro de los Ríos. 21 de abril de 1621].

Licencia del Ordinario [Madrid, 26 de agosto de 1620, ante Eugenio López, notario].

f. 3r. Aprovacion del / padre fray Prudencio de Luzon, / Abad del Monasterio de Santa Maria de Armentera, de la Orden / de S. Bernardo.

f. 3i. CENSVRA DE LOPE / re Vega Carpio.

Por mandado de V. Alteza he visto este li-/bro de la Iusta Poetica, que se hizo a las / fiestas del Santísimo Sacramento en la villa / de Cifuentes, y no hallo en el cosa opuesta / a nuestra Santa $\mathrm{Fe}$, y buenas costumbres; an-/tes cosas de mucho ingenio, porque merece / que V. Alteza le de, siendo seruido, la licencia / que pide; para que con este exemplo se ani-/ men los deuotos a celebrar este dia, y tengan / premios de honra los que se exercitan en su / alabahça. Este es mi parecer, en Madrid a dos / de Setiembre, de 1620 .

Lope de Verga Carpio.

f. 4r-5r. Al lector.

f. 38r-40r. Octavas del Lcdo. Juan de Orozco.

Colofón: En Madrid, / Por Tomas Iunti, Impressor del Rey nuestro señor. / Año de M.DC.XXI.

3. Este es el libro que en 1935 existía en la Facultad de Letras de Madrid 2. La aprobación fue transcrita por Zamora Lucas $^{3}$; sin embargo,

1 El Dr. Juan Cutiérrez de Solórzano. comnilador de los textos, nació en Cifuentes, alrededor de 1560 ; en 1592 se doctoró en medicina por Alcalá, fue conocido de Calderón y médico de Su Majestad (1632) y de la Inquisición (1636). Murió con más de ochenta años, pues su testamento es de 1642. Zamora LuCAS, Lope de Vega, censor de libros, pp. 98-99.

a Vid., arriba, nota 2 y referencia del texto.

8 Lope de Vega, censor de libros, núm. 30, p. 48. 
no la décima, que puedo completar ${ }^{1}$. Mide $19,8 \mathrm{cms}$. de alto por 14 de ancho.

NAVE / TRAGICA / DE LA INDIA DE / PORTUGAL, / POR FRANCISCO DE CONTRERAS, / Natural de Argamasilla de Alba. / A LOPE FELIX DE VEGA CARPIO, / Fiscal de la Camara- Apostolica. / [Viñeta geométrica] / CON LICENCIA / En Madrid, por Luis Sánchez, año de 1624.

f. 2r. [sin numerar] El Doctor Mira de Mescua / A la nave tragica. En éste $\mathrm{y}$ en $2 v^{\prime}$, hay elogios de don Rodrigo de Galiana, don Luis Ferreira Un amigo del autor, don Ioseph de Samano.

f. 3r. Lope Felix de Vega / Carpio / A L A U T OR /

La Naue que a Portugal

Dio llanto en vez de tesoro,

Contreras con lineas de oro

Buelue en Argos celestial:

Oy como el Aue Imperial

La sube en plumas tan bellas,

Que atreuida al Sol con ellas

Tragica dexa de ser:

Que su ingenio pudo hazer

Todos sus versos estrellas.

A continuación, elogio de don Pedro Ypenarieta.

f. 3v. A LOPE DE VEGA / Carpio.

4. A pesar de lo que se dice en la portada, y repiten los bibliógrafos, la carta de Lope de Vega no existe. El ejemplar que manejo tiene una página reproducida; es posible que los restauradores no se dieran cuenta de que falta la respuesta del Fénix o que ésta no haya existido nunca ${ }^{2}$. Es

1 Millé, op. cit., p. 400, dio las siguientes indicaciones:

(95. CONTRERAS (FRANCISCO DE), Nave trágica, Madrid, 1624. Aprobación de Lope, Madrid, 12 de noviembre de 1624. Décima de Lope al autor (PÉrez PAstor, $B$. $M$., III, núm. 2.055).ఎ Añádase: Gallardo, II, 561-562. Sobre la enigmática persona de Francisco de Contreras, vid. Zamora, Lope, censor, pp. 80-81. Allá se insiste en que la décima es «desconocida para los éruditos».

2 Zamora Lucas, Lope, censor, pp. 82-83, da curiosos informes sobre el opúsculo: al parecer no existen, ejemplares en España, pero había un ejemplar en Portugal (1397) que perteneció al bibliógrafo José María Nepomuceno. De él lo adquirió el Marqués de Jerez de los Caballeros y, más tarde, la biblioteca, del noble español «fue vendida a América, donde probabemente existirá el ejemplar del Elogio ignorándose la ciudad y Bibliotecan. Como es sabido la Hispanic Society adquirió, en 1902, la biblioteca de Jerez de los Caballeros, y, por ello, puedo añadir el último eslabón a la cadena. (Vid. 
un folleto de cuatro páginas sin numeraw y 16 folios con numeración; nirfe 20 cms. de alto por 13,8 de ancho ${ }^{1}$.

ELOGIO / DE POETAS / LVSITANOS / AL FENIX DE ESPAÑA Fr LOPE / Felix de Vega Carpio, en su laurel / de Apolo / POR EL ALFERES JACINTO / Cordero, con ona carta en respuesta al / Autor, del mismo Fenix de / España / DIRIGIDo a LA SENora doNa / Cicilia de Meneses / Año [riñeta lineal] 1631 / EN LISBOA / Con todas las licencias necessarias, / por Iorge Rodriguez.

5. Del conocido novelista Alonso del Castillo Solórzano no escaseai los elogios a Lope ${ }^{2}$ y sabemos su amistad con Montalbán. Sin embargo, y aunque la obra que voy a describir debía ser conocida, muy pocos la pudieron ver ${ }^{3}$. Porque sorprende que traiga a colación que, gracias a ella, sepamos que Castillo Solórzano era calvo y que no se utilicen otras referencias a que haré mención. Que el libro no ha debido ser muy asequible se explica por la nota manuscrita que hay en el ejemplar que manejo: «libro rarísimo, por haberse perdido el barco que los traía de Italia. Sólo se salvaron los que quedaron allá». El volumen consta de 320 páginas y mide $16 \mathrm{cms}$. de alto por 10 de ancho.

FAVORES DE LAS MVSAS / Hechos a Don Sebastian / Francisco de Medrano. / En varias Rimas y Comedias, que compuso en la mas / celebre Academia de Madrid donde fue / Presidente meritissimo. / Recopilados por Don Alonso de Castillo / Solorzano intimo amigo del Auctor. / AL EMINENTISS. Y ESCELL. SENOOR EL / Señor Theodoro Triuultio Diacono Cardenal de / la S. Iglesia Romana del Titulo de S. Cesareo, / y legado à latere de la S. Sede Apóstolica y / de muestro Reatiss. Señor Vrbano Octauo / en la prouincia de la Marca de Anco-/na Vicario General en lo spiritual, / y temporal del Sacro Romano / Imperio Principe de Misocco, / y de Val Misolcino. Conde / de Meleio y Gorgonzo-/la Señor de Cotoño, y / Vengazuelo / [adorno] / CON PRIviregro / En Milan, por I naptista Malatesta Impressor / Regio, y Ducal, acosta de Carlo Ferranti Librero. / año 1631. Con licencia de los Superiores.

F. Rodríguez Marín, Ei fundador de The Hispanic Society of America, apud Burla burlando, Madrid, 1914, citado por A. Ronríguez-MoÑino, Los pliegos poéticos de la Coleción del Marqués de Morbecq. Madrid, 1962, p. 36).

La seguridad de que el ejemplar de la Hispanic Society es el mismo que el de Nepomuceno está confirmada porque en el catálogo del bibliófilo portugués se dice: Front. feito a penna, y el ejemplar que yo manejo tiene - también- rehecha la portada.

I ZaMora LUCAS, Lope, censor, p. 82, ha reunido algunos datos sobre Jacinto Cordeiro: nació en Lisboa, donde murió (1646) a los cuarenta años; está enterrado en la parroquia de Santa María Magdalena de la capital portuguesa.

2 Vid. Zamora, Lope, censor, p. 76.

I Ib., p. 75 . 
[f. 1v.] ${ }^{1}$ Indice.

[f. 2r.] Aprobación de la Inquisición, por Fr. Bartolomé Corradi, O. P.

[f. 2 $\pi$.] Aprobación del ordinario.

[f. $3 r-v$.] Dedicatoria a la Inquisición del Estado de Milán.

[f. 4r.] Carlo Ferranti Libraro, Alli lettori.

[f. 4v-6r.] E1 Dr. D. Sebastián Francisco de Medrano a D. Alonso Castillo de Solórzano. En el $4 v$, se lee:

"Pero quando fuy conociendo poco a poco la prudencia, y reconoci a vn Lope de Vega honrra de mi Patria, milagro de nuestra nacion, y prodigio para las estrañas, a quien todos los que quisieran confessar la verdad, sauen la enseñança que le deben.»

En el $5 r$ se confiesa condiscípulo y amigo de Pérez de Montalbán.

[f. $6 z^{-}-7 r$.] Epístola al que leyere.

[f. 7v.] Dedicatoria.

p. 1-20. Primer fauor en lo epico o heroyco por Caliope.

p. 21. Las Venganzas de Amor, comedia [mitológica] en tres actos.

p. 108. Segundo favor... por la musa Clio en lo elegiaco. Libro segundo de sus obras.

p. 117. Tercero favor... por la musa Melpomene en lo Tragico. Libro tercero de sus obras.

p. 119. El Lvzero eclipsado, historia tragica [de San Juan Baustista], en cinco actos.

p. 180. . Quarto favor... por la Mussa Erato en lo jocoso o burlesco.

p. 191. Lealtad, amor y amistad [comedia en tres actos].

p. 293. Trivmpho de la alegria, en la venida de mi señora doña Francisca de Cordoba.

p. 314.318. Elogio por don Juan Baptista Ottho.

En la p. 319 , se dice que el t. II comprendería:

El sexto favor (Terpsichore, con la Comedia La Luna de Valencia).

E1 séptimo, de lo satyrico, por Polimnia.

El octavo, de lo Bucolico, por Euterpre, "con la comedia pastoral Mal propio por bien ajeno, y dos eglogas".

El noveno, de lo divino, por Urania, con la Comedia de San Agustín titulada Lo que las lagrimas pueden.

Manuel Alvar.

\section{Universialad de Granada.}

L La foliación entre paréntesis cuadrados es mía, a falta de otras referencias en el texto. 